ARTICLE

DOI: $10.1038 / \mathrm{s} 41467-018-04080-4$

\title{
Chiral Landau levels in Weyl semimetal NbAs with multiple topological carriers
}

Xiang Yuan 1,2, Zhongbo Yan ${ }^{3}$, Chaoyu Song ${ }^{1,2}$, Mengyao Zhang ${ }^{4,5}$, Zhilin Li ${ }^{5,6}$, Cheng Zhang (1) 1,2, Yanwen Liu ${ }^{1,2}$, Weiyi Wang ${ }^{1,2}$, Minhao Zhao ${ }^{1,2}$, Zehao Lin'1,2, Tian Xie ${ }^{1,2}$, Jonathan Ludwig7, Yuxuan Jiang ${ }^{7}$, Xiaoxing Zhang ${ }^{8}$, Cui Shang ${ }^{8}$, Zefang $Y e^{1,2}$, Jiaxiang Wang ${ }^{1,2}$, Feng Chen ${ }^{1,2}$, Zhengcai Xia ${ }^{8}$, Dmitry Smirnov ${ }^{7}$, Xiaolong Chen ${ }^{5,6}$, Zhong Wang (i) ${ }^{3,6}$, Hugen $\operatorname{Yan}^{1,2}$ \& Faxian Xiu ${ }^{1,2,9}$

Recently, Weyl semimetals have been experimentally discovered in both inversionsymmetry-breaking and time-reversal-symmetry-breaking crystals. The non-trivial topology in Weyl semimetals can manifest itself with exotic phenomena, which have been extensively investigated by photoemission and transport measurements. Despite the numerous experimental efforts on Fermi arcs and chiral anomaly, the existence of unconventional zeroth Landau levels, as a unique hallmark of Weyl fermions, which is highly related to chiral anomaly, remains elusive owing to the stringent experimental requirements. Here, we report the magneto-optical study of Landau quantization in Weyl semimetal NbAs. High magnetic fields drive the system toward the quantum limit, which leads to the observation of zeroth chiral Landau levels in two inequivalent Weyl nodes. As compared to other Landau levels, the zeroth chiral Landau level exhibits a distinct linear dispersion in magnetic field direction and allows the optical transitions without the limitation of zero $z$ momentum or $\sqrt{B}$ magnetic field evolution. The magnetic field dependence of the zeroth Landau levels further verifies the predicted particle-hole asymmetry of the Weyl cones. Meanwhile, the optical transitions from the normal Landau levels exhibit the coexistence of multiple carriers including an unexpected massive Dirac fermion, pointing to a more complex topological nature in inversion-symmetrybreaking Weyl semimetals. Our results provide insights into the Landau quantization of Weyl fermions and demonstrate an effective tool for studying complex topological systems.

\footnotetext{
${ }^{1}$ State Key Laboratory of Surface Physics and Department of Physics, Fudan University, 200433 Shanghai, China. ${ }^{2}$ Collaborative Innovation Center of Advanced Microstructures, Fudan University, 200433 Shanghai, China. ${ }^{3}$ Institute for Advanced Study, Tsinghua University, 100084 Beijing, China.

${ }^{4}$ International Center for Quantum Materials, School of Physics, Peking University, 100871 Beijing, China. ${ }^{5}$ Beijing National Laboratory for Condensed Matter Physics, Institute of Physics, Chinese Academy of Sciences, 100190 Beijing, China. ${ }^{6}$ Collaborative Innovation Center of Quantum Matter, 100871 Beijing, China. ${ }^{7}$ National High Magnetic Field Laboratory, Tallahassee, Florida 32310, USA. ${ }^{8}$ Wuhan National High Magnetic Field Center, Huazhong University of Science and Technology, 430074 Wuhan, China. ${ }^{9}$ Institute for Nanoelectronic Devices and Quantum Computing, Fudan University, 200433 Shanghai, China. These authors contributed equally: Xiang Yuan, Zhongbo Yan, Chaoyu Song, Faxian Xiu. Correspondence and requests for materials should be addressed to H.Y. (email: Hgyan@fudan.edu.cn) or to F.X. (email: Faxian@fudan.edu.cn)
} 
$\mathrm{D}$ irac semimetals can be viewed as special Weyl semimetals with spin degeneracy ${ }^{1}$. The lifting of the Weyl nodes degeneracy can be accessed by breaking inversion symmetry (IS) in Dirac semimetals. It was recently realized in non-centrosymmetric compounds $\mathrm{NbAs}$ family ${ }^{1-13}$. Such a Weyl semimetal phase is featured by Fermi arcs and chiral anomaly, both of which were intensively investigated ${ }^{6-16}$. The Fermi arcs with unique spin texture have been observed by photoemission on the surfaces of Weyl semimetals $7,910,12-14$. The open-contour feature distinguishes themselves from traditional surface states and results in novel cyclotron orbits under magnetic fields ${ }^{15}$. Meanwhile, the predicted chiral or Adler-Bell-Jackiw anomaly, a chirality imbalance phenomenon in Weyl semimetals, has been widely observed ${ }^{16-24}$, although the origin of some experiments is still under debate ${ }^{25,26}$. Besides the Fermi arcs and chiral anomaly, one of the most important features of Weyl semimetals is the presence of the zeroth chiral Landau level, which is linearly dispersed in $K_{z}$ direction ${ }^{27-29}$. It was theoretically predicted to be a hallmark of Weyl fermions that leads to the widely observed chiral anomaly in Weyl semimetals ${ }^{16-22,24}$. However, directly detecting this distinct zeroth Landau level through conventional photoemission or transport experiments remains an unprecedented challenge since both magnetic field and Landau level spectroscopy are required ${ }^{27}$. Also, experimentally approaching the quantum limit is strictly demanded to distinguish the chiral Landau level from other degenerated Landau levels.

Here, we investigate the quasiparticle dynamics in Weyl semimetal $\mathrm{NbAs}$ by means of magneto-optics. The ability to detect the Landau levels via the optical reflectance under high magnetic fields enables the observation of the zeroth chiral Landau levels and complex topological nature near the quantum limit. Two sets of inequivalent Weyl cones with different Fermi velocity were identified through $\sqrt{B}$ and $\sqrt{n}$ dependence of the optical transition frequency. The intra-band transition edges were observed as a direct consequence of the chiral Landau levels in both of inequivalent Weyl nodes. The dramatic distinction from other Landau levels, however, is the linear dispersion in $k_{z}$ (magnetic field) direction, resulting in a unique field evolution of transition energy. Detailed study of the transitions between the chiral and normal Landau levels reveals a large particle-hole asymmetry in Weyl cones with higher Fermi velocity which agrees well with the predictions from first-principle calculations ${ }^{30}$. Finite scattering helps to enhance the feature from transition edge ${ }^{27}$. Also, corroborated with our theory, five kinds of optical transitions involving the chiral and conventional Landau levels are unambiguously distinguished, which construct the whole band structure of $\mathrm{NbAs}$ under magnetic field as summarized in the end. Besides these two inequivalent Weyl nodes, we also reveal the coexistence of massive Dirac fermions, as well as trivial band insulating state, suggesting a more complex topological nature in the Weyl semimetal family. The comparison among different optical transitions in NbAs exhibits the uniqueness of the chiral Landau levels. In the following, we will describe these five optical transitions and distinguish their origins one by one, which show the clear contribution from the chiral Landau levels. The comprehensive study of Landau level spectroscopy in NbAs provides insights into multiple novel topological carriers and constitutes an ideal experimental tool for studying complex quasi-particles with their own topological nature.

\section{Results}

Single-crystal NbAs. NbAs crystalizes in body-centered tetragonal unit cell with space group $\mathrm{I}_{1} \mathrm{md}$ and point group $\mathrm{C}_{4 \mathrm{~V}}{ }^{31}$, as shown in Fig. 1a. Since this type of structure lacks a horizontal mirror plane, the IS in NbAs is naturally broken, which leads to the lift of the spin degeneracy, necessary for developing the Weyl semimetal phase. Ab initio calculations predict 24 Weyl nodes in the first Brillouin zone of $\mathrm{NbAs}^{1,7}$, where they always come in pairs with opposite chiralities or monopole charges. The studied single crystals were grown by transport method ${ }^{32}$ and their crystalline orientation and chemical ratio were examined by X-ray diffraction (XRD) and electrondispersive X-ray spectroscopy (EDX), respectively (Fig. $1 \mathrm{~b}$ and Supplementary Figure 1). Three prominent peaks can be clearly identified as (004), (008), and (0012) crystal planes ${ }^{31,33}$. The lattice constant is calculated to be $a=3.45 \AA$ and $c=11.68 \AA$ which is consistent with the previous studies ${ }^{31}$. The stoichiometry of the sample confirmed by the EDX ensures the comparatively low Fermi level and less undesired defects ${ }^{34}$.

Magneto-optical spectrum. Magneto-infrared measurements have been proved to be an effective tool for study Landau
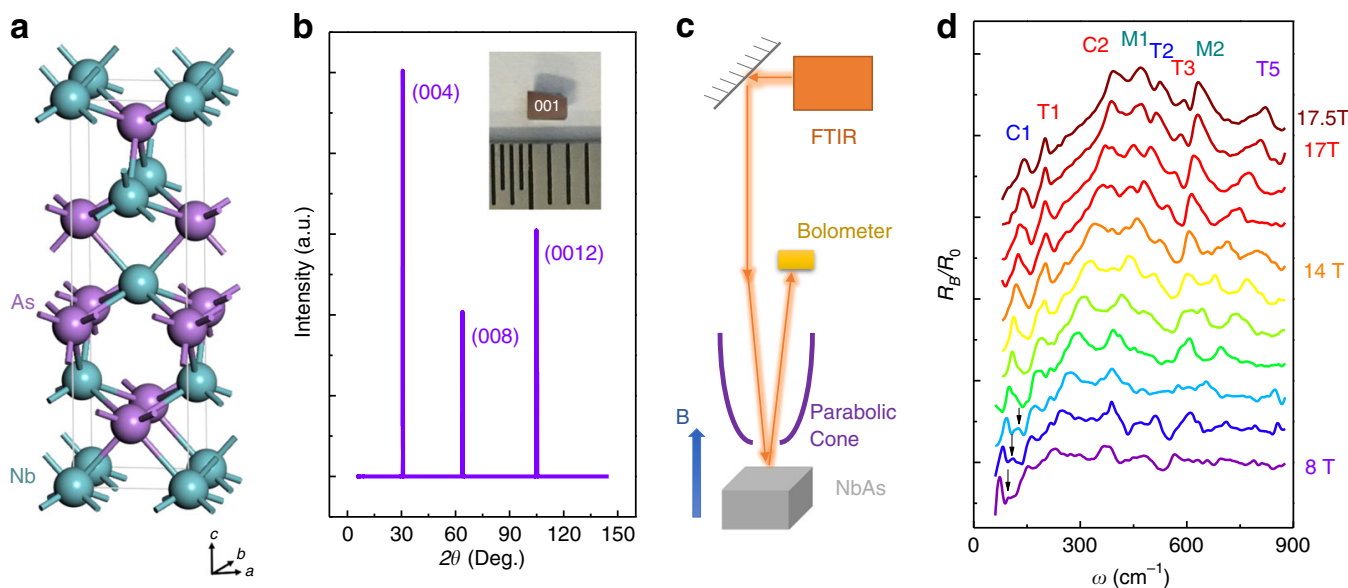

Fig. 1 Material characterizations and magneto-optical spectra. a Body-centered tetragonal structure of NbAs with inversion-symmetry-breaking. b Singlecrystal XRD spectrum, showing the (001) crystal planes of single crystallinity of NbAs. The inset is a photo of the sample with a length of 2 mm. c Schematic plot of the magneto-optical experimental setup. $\mathbf{d}$ Magneto-optical spectra of NbAs under different magnetic fields. The reflection rate under magnetic field versus photon energy is normalized by zero-field reflection measured from the same setup. The curves for different magnetic field are vertically shifted for clarity. These features with different magnetic field evolution are classified as C-type, M-type, and T-type. Note that the dark arrows denote C3 transitions 
a

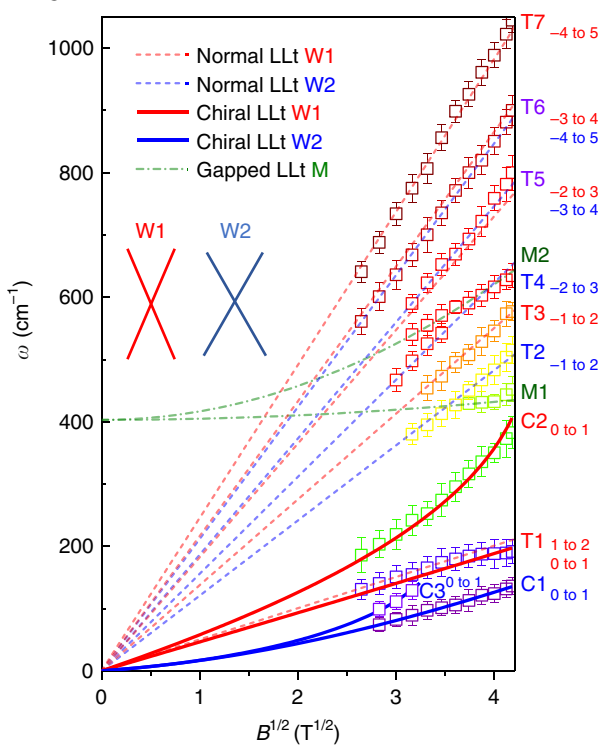

b

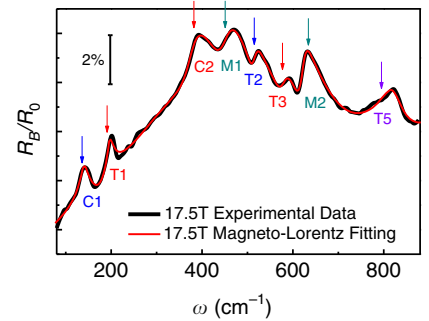

d

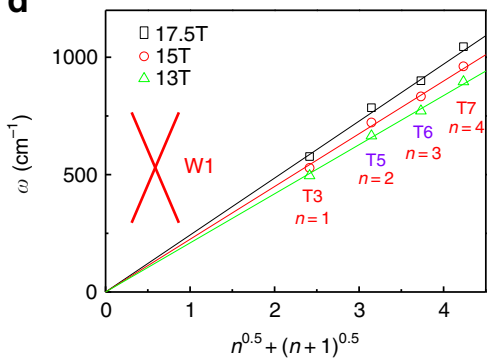

c

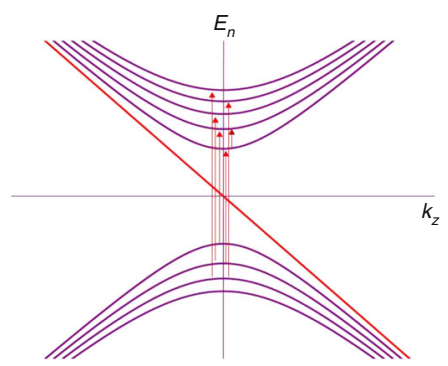

e

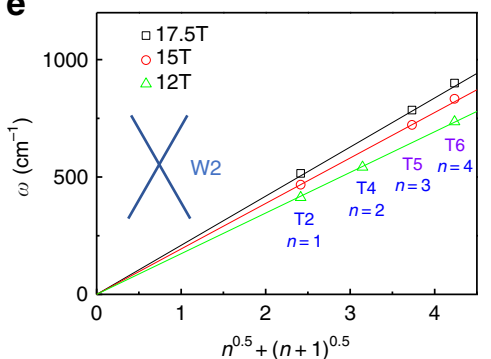

Fig. 2 Inter-Landau-level transition energy from multiple carriers. a Transition energy versus $\sqrt{B}$ in the far-infrared range. Colored lines and labels are used to differentiate the origin of each feature. Red and blue dashed straight lines are guidelines for T-type transitions pointing to the origin of the plot, indicative of the two inequivalent massless Weyl fermions. Dark green dashed lines are fitting curves with linear-in- $B$ evolution for $M$-type transitions, suggesting gapped electronic bands with band inversion. Red and blue solid lines are fitting curves for the intra-band transition involving the zeroth chiral Landau levels in Weyl nodes. In this plot, colors in red, blue, dark green (for curve, guidelines and labels) denote the contributions from Weyl node 1 (W1), Weyl node 2 (W2), and gapped band, respectively. Inset shows that W1 has a higher Fermi velocity than W2. Labels in purple present that the feature is contributed by both Weyl nodes, thus mixing red and blue. The label $-n \rightarrow n+1$ denotes the initial and final Landau levels involved in the optical transition process. $-(n+1) \rightarrow n$ transition is also involved but not labeled for simplicity. Error bars are added based on both experimental resolution and fitting uncertainty. $\mathbf{b}$ The normalized reflection data from experiments (black) and the Magneto-Lorentz fitting (red). The energy of the inter-Landau level transition in $\mathbf{a}$ is described by the fitted energy (arrows) instead of apparent peak maxima. $2 \%$ is the change of the reflection under magnetic field. c Landau level dispersion of massless Weyl fermion in NbAs. Only the lowest nine branches are plotted for illustration. The arrows delineate the allowed optical inter-band transition processes between Landau levels at $k_{z}=0$. d, e Fan diagrams for W1 and W2 under different magnetic fields. Solid lines are guidelines pointing to the origin of the plot, indicative of the massless Weyl fermions and verifying the assignment of transition index. Labels indicate the transition and Landau level index

quantization for topological materials that can provide rich information, such as band parameters and field response ${ }^{35}$. The measurements of magneto-infrared spectroscopy are combined with magneto-transport, where the important parameters are extracted that help to understand Landau levels. Here we take magneto-optical spectrum on the (001) crystal surface of NbAs as shown in Fig. 1b inset. The schematic experimental setup of the magneto-optical measurements is displayed in Fig. 1c. Faraday geometry were used in a superconducting magnet with the magnetic field up to $17.5 \mathrm{~T}$. More experimental details are available in the method section. As shown in Fig. 1d, the reflectivity spectra were normalized by the zero-field data. A series of reflection features can be resolved. The reflection maxima and minima systematically shift towards the higher energy with increasing magnetic field. Given the high mobility of $\mathrm{NbAs}$ at low temperatures, the field-dependent peaks can be assigned to the Landau level transitions. Although all of the peak energies increase with field, certain differences are discernible. In Figs 1d and $2 \mathrm{a}$, some of the peak energies increase linearly with the magnetic field (denoted as M-type in dark green) while the others do not. The observed difference indicates the distinct origins of low-energy excitations in the system. For a classical system with a quadratic energy dispersion $\varepsilon=\hbar^{2} k^{2} / 2 m^{\star}$, the Landau quantization under magnetic field reads

$$
E_{n}=(n+1 / 2) \hbar e B / m *,
$$

where $n, e, \hbar$ represent the Landau index, the elementary charge and the reduced Plank's constant, respectively. Optical selection rules require $\Delta n=\left|n_{i}\right|-\left|n_{f}\right|= \pm 1$, where $n_{i}, n_{f}$ denote the
Landau level index of the initial and final states. The Landau level transition with the T-type and C-type features are in stark contrast to the classical picture, while the M-type peaks fit well to the linear-in- $B$ rule (Fig. 2a). The notations $\mathrm{C} / \mathrm{T} / \mathrm{M}$ are named after several terms: Chiral Landau level, normal Transition, and Multiple bands, which will be discussed in the following sections. All these salient features are reproducible as demonstrated in Supplementary Note II from another NbAs sample of the same batch with an identical growth condition.

Origin of the inter-Landau-level transitions. To quantitatively study the spectrum and understand the origin of each transition, we extracted the frequency of inter-Landau-level transitions and plotted it against the magnetic field in Fig. 2a. Generally, Landau level transition induces a peak in the optical conductivity. In order to extrapolate the transition energy, an absolute reflection spectrum with gold overcoating technique is required (Supplementary Note III). Then the experimental data were fitted to the Drude-Lorentz model (Supplementary Note X) in the presence of magnetic field ${ }^{36-39}$. The fitted curve in Fig. $2 b$ shows a good consistency with the original experimental data for all magnetic fields (Supplementary Figure 8 in Supplementary Note X). The extracted transition energy typically locates nearby but with a lower energy than where the apparent peak is positioned in the original data (especially for T-type and M-type transitions). The inter-Landau-level transition energy in Fig. 2a is obtained from the aforementioned fitting scheme.

Fan diagram reveals rich features that can be used to determine the electronic structure of NbAs under magnetic fields. A series of 

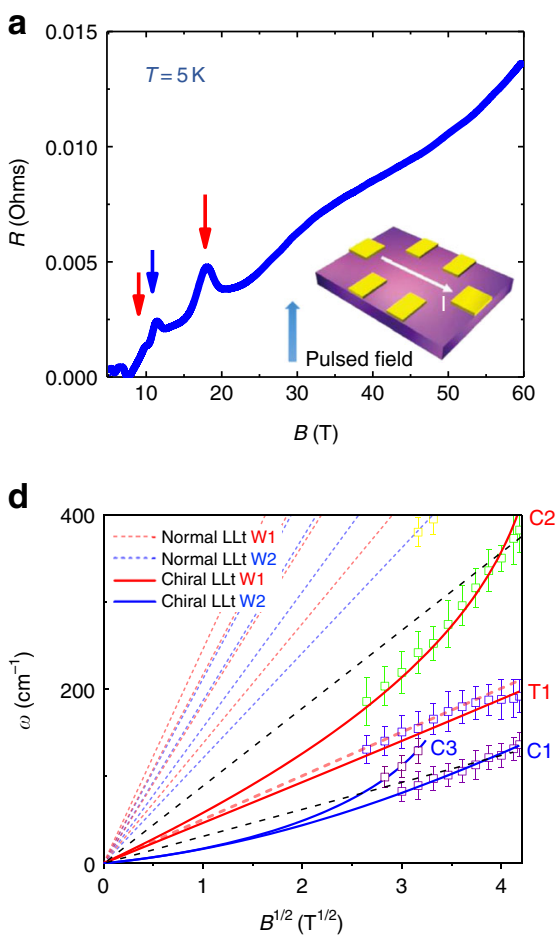
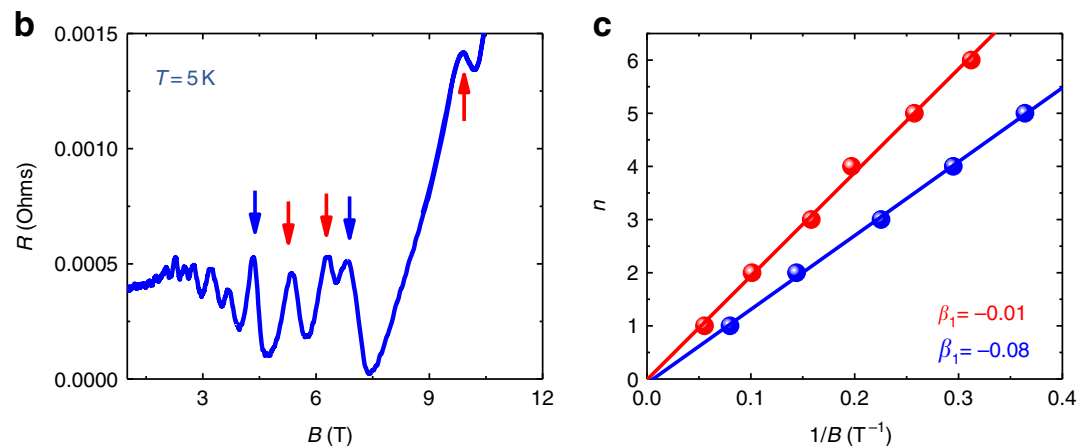

e

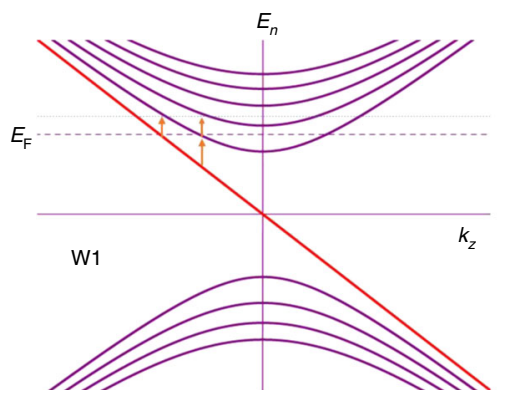

$\mathbf{f}$

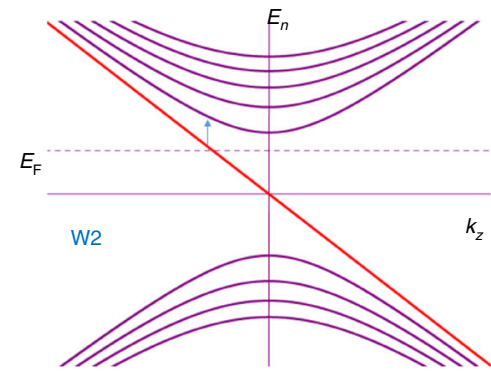

Fig. 3 Quantum limit and chiral Landau levels. a, b Longitudinal magneto-resistance with magnetic field up to $60 \mathrm{~T}$ and $10 \mathrm{~T}$, respectively. The quantum limit is reached at $12 \mathrm{~T}$ and $19 \mathrm{~T}$ for two Weyl nodes. The inset shows the schematic drawing of the experimental setup. Arrows represent the peak position. The colors of the arrows denote the oscillation components from two inequivalent Weyl nodes. c Landau fan diagram. Landau index is plotted against $1 / B$. Resistivity peak represents the integer Landau index. The observed intercept suggests non-trivial topological nature of both components in the SdH oscillations. d Inter-Landau-level transition in terahertz range where the intra-band transition is allowed. Red and blue lines have the same meaning as Fig. 2a. Black dashed lines are guidelines (linear in $\sqrt{B}$ ), showing a deviation of chiral Landau level transitions from the normal ones. The fitting curve indicates the origin of $\mathrm{C} 1 / \mathrm{C} 3$ and $\mathrm{C} 2$ from the zeroth chiral Landau level of W2 and W1, respectively. e, f Schematic plots of Landau level dispersion for W1 and W2. The chiral Landau level is linearly dispersed. For W1 (not reaching the quantum limit), there exists two intra-band transition edges while W2, already in the quantum limit $(B>12 \mathrm{~T})$, has only one

transition energies follow a straight line pointing to the origin of the plot on a scale of $\sqrt{B}$, which provides key information for the T-type transitions as shown in Fig. 2a. Massless Weyl fermions are described by the Hamiltonian $H(\mathbf{k})=v_{\mathrm{F}} \mathbf{k} \cdot \sigma \quad(\hbar=1$ for notation simplicity) where $\sigma=\left(\sigma_{x}, \sigma_{y}, \sigma_{z}\right)$ and $v_{\mathrm{F}}$ represent the Pauli matrices and the Fermi velocity, respectively. With the introduction of a magnetic field along $z$ direction, the formed Landau levels read ${ }^{18}$,

$$
E_{n}\left(k_{z}\right)=\left\{\begin{array}{c}
\operatorname{sgn}(n) v_{\mathrm{F}} \sqrt{k_{z}^{2}+2|n| e B},|n| \geq 1 \\
-v_{\mathrm{F}} k_{z} n=0
\end{array}\right.
$$

The lowest Landau level is of chiral nature which makes it quite different from the higher Landau levels. Based on Eq. (2), it is readily found that the density of states is given by

$$
D(\varepsilon)=\frac{e B}{4 \pi^{2} v_{\mathrm{F}}}\left[1+2 \sum_{n=1} \Re\left(\frac{|\varepsilon|}{\sqrt{\varepsilon^{2}-2|n| e B v^{2}}}\right)\right] .
$$

$\Sigma$ is a sum symbol. From Eq. (3) and Fig. 2c, the singularity occurs at $k_{z}=0$ where the density of state becomes infinite. Therefore, the optical transition energy should follow $\sqrt{B}$ and $\sqrt{n}$ law for Weyl semimetals with non-zero index. Experimentally, all the T-type transitions clearly adopt the $\sqrt{B}$ dependence, indicating the Landau level transition from non-zero index (illustrated by red arrows in Fig. 2c), and thus serving as a direct evidence of ultra-relativistic fermions ${ }^{29,40-43}$. Note that all the schematic plots of Landau level dispersion are also applicable to the hole-doped Weyl semimetals because the optical transition is schematically symmetric, i.e., it has the same absorption energy for the electron/hole doping with the same carrier density.

We now assign the specific Landau level index $n$ and verify the $\sqrt{n}$ dependence. Deduced from Eq. (2) and the optical selection rules, the resonance frequency ${ }^{42}$ or the slope of T1-T7 in Fig. 2a, designated as $S 1-S 7$, should be proportional to $\sqrt{n+1} \pm \sqrt{n}$, where the positive (negative) sign is for inter-band (intra-band) transition. Apparently, T1-T7 do not simply follow this rule and the observed optical transition cannot be explained by the Landau quantization of a single ideal Weyl cone. In fact, we have noticed that the ratio of the adjacent slopes keeps nearly a constant, i.e., $S 7 / S 6 \approx S 6 / S 5 \approx S 5 / S 4 \approx S 3 / S 2$. This clearly shows signatures of two inequivalent Weyl nodes. By further calculating $S 7: S 6: S 5: S 3 \approx(\sqrt{4}+\sqrt{5}):(\sqrt{3}+\sqrt{4})$ : $(\sqrt{2}+\sqrt{3}):(\sqrt{1}+\sqrt{2})$, these four transitions can be identified to originate from one Weyl node, denoted as Weyl node 1 (W1). Then the peak assignments can be made as follows: T3 corresponds to $L_{-1} \rightarrow L_{2}$ or $L_{-2} \rightarrow L_{1}$ and $\mathrm{T} 5 L_{-2} \rightarrow L_{3}$ or $L_{-3} \rightarrow L_{2}$, and so on so forth for T6 and T7. Here $L_{n}$ represents the $n^{\text {th }}$ Landau level. Note that only $L_{-n} \rightarrow L_{n+1}$ is presented with labels for simplicity. These assignments can be further verified by the fan diagram in Fig. 2d, where $\sqrt{n+1}+\sqrt{n}$ dependence can be clearly seen. The assignments for the second Weyl node (W2, Fig. 2e) involving T6, T5, T4, and T2 can be made in the same manner as that for $\mathrm{W} 1$. Therefore, we elucidate the existence of two inequivalent Weyl nodes and the Landau level energy is indeed proportional to $\sqrt{B}$ and $\sqrt{n}$. By performing a linear fit, the 
Fermi velocity is derived to be $v_{\mathrm{F} 1}=2.0 \times 10^{5} \mathrm{~m} / \mathrm{s}$ and $v_{\mathrm{F} 2}=1.7 \times$ $10^{5} \mathrm{~m} / \mathrm{s}$, which matches well with the previous first-principle calculations $s^{30}$. Weyl nodes 1 and 2 are illustrated in red and blue in Fig. 2, respectively. It is worth mentioning that T5 and T6 include the transitions from both Weyl nodes and, therefore, are labeled in purple. This overlapping is a natural consequence of two inequivalent Weyl cones as shown in Supplementary Note X. Usually for the system close to quantum limit, the inter-band transition

with higher index should show smaller amplitude. Here the comparatively higher amplitude of T5, T6 can be explained by the overlapping of these close transitions. The larger width of T5 and T6 originates from same mechanism. To distinguish more clearly how those different electronic bands influence the optical transitions, Fig. 2a is replotted and divided into several panels for further clarification in Supplementary Note IV and Supplementary Figure 4. All the observed features originated from different bands are also summarized in the end.

Quantum limit for inequivalent Weyl nodes. The existence of resonant peaks corresponding to $L_{-1} \rightarrow L_{2}$ or $L_{-2} \rightarrow L_{1}$ suggests that the system is close to the quantum limit. The Fermi velocity and the energy offset between two inequivalent Weyl nodes are also suggested by angle-resolved photoemission spectroscopy 9 . Different from inter-band transitions, which always come in pairs (blue and red for two Weyl nodes), as shown in Fig. 2a in the low frequency regime, there exists only one linear-in- $\sqrt{B}$ transition (T1), which leads us to check the position of Fermi level and the quantum limit. Therefore, magneto-transport measurements were carried out with a Hall-bar device on the same sample with the same geometry as the magneto-optical measurements. Figures. 3a, $\mathrm{b}$ present the longitudinal MR in different field regimes. Clear Shubnikov-de Haas (SdH) oscillations can be resolved on a large upward background. The ultimate quantum limit is reached near a field of $18 \mathrm{~T}$ similar to the previous magnetic torque measurements $^{15}$. A large MR reaches $60,000 \%$, which features the compensated material and topological massless fermion with the suppressed backscattering ${ }^{44}$. We extracted the peak positions of the resistivity and assigned the integer index. Two sets of $\mathrm{SdH}$ oscillations were found as shown in Fig. 3c (also, arrows in different colors in Fig. 3a, b). It is noted that the closed cyclotron orbit under magnetic field follows the Lifshitz-Onsager quantization rule $S_{\mathrm{F}} \frac{\hbar}{e B}=2 \pi\left(n+\frac{1-\phi_{\mathrm{B}}}{2}\right)$, where $\mathrm{S}_{\mathrm{F}}, \phi_{\mathrm{B}}$ are Fermi surface area and Berry phase, respectively. Performing the linear fit in the fan diagram yields the intercepts, which are nearly zero, indicative of the topological non-trivial Berry phase. The non-trivial topology of the oscillation components evidences two gapless Weyl nodes. Consistent with the previous reports ${ }^{45,46}$, our Hall effect measurements suggest a typical two-carrier transport behavior (Supplementary Note V and Supplementary Figure 5) and the quantum limit is reached with magnetic field $19 \mathrm{~T}$ and 12 $\mathrm{T}$ for the inequivalent Weyl nodes. The fact that one of the intraband transitions is missing can now be explained by the entrance of the quantum limit.

Chiral Landau levels. It has a profound influence on intra-band transitions when the system reaches the quantum limit. As shown in Fig. 3d, different from the discussed T-type or M-type transitions, there remains three other peaks denoted as $C 1$ to $C 3$, which follow neither linear-in- $B$ nor linear-in- $\sqrt{B}$. Since all the allowed transitions with non-zero index have been exclusively identified at high energy, the remaining $\mathrm{C} 1$ to $\mathrm{C} 3$ are anticipated to come from the intra-band transitions between chiral $L_{0}$ and
$L_{ \pm 1}$. The linearly dispersed zeroth Landau level in Weyl semimetal is unique compared with traditional systems. It has been used to explain the four-fold splitting in magneto-optical spectrum of $\mathrm{ZrTe}_{5}$ and was further studied by circular polarization resolved experiments ${ }^{42,47}$. It also differs from other topological materials such as Kane semimetal $\mathrm{HgCdTe}^{48}$ or Dirac semimetal graphene $e^{49}$, where a flat zeroth Landau level along $k_{z}$ locates at zero energy regardless of various magnetic fields, resulting in an energy gap between the zeroth and the first Landau level. A previous magneto-optical study also suggests $\mathrm{Cd}_{3} \mathrm{As}_{2}$ to be Kane semimetal partly due to the lack of chiral Landau level ${ }^{29}$. The transitions involving the zeroth Landau level should occur at $k_{z}=0$ in Kane or 2D Dirac semimetals while a fixed zeroth Landau level should result in a linear-in- $\sqrt{B}$ relation for $L_{0} \rightarrow L_{1}{ }^{43,42,4849}$, As to Weyl semimetals, the 3D structure with Weyl cones ensures a different form of zeroth Landau level, as described by Eq. (2), with unique optical features that agree well with the observed C-type transitions in the following four aspects. Firstly, for the lowest energy intra-band transition, it does not necessarily occur at $k_{z}=0$ due to the lack of density of state maxima. Instead, the allowed transition has a certain frequency edge where the density of states experiences a sudden increase from zero when the Fermi level crosses the zeroth Landau level as shown in Fig. 3e, $\mathrm{f}$ (colored arrows). Another fact that needs to be pointed out is that in the presence of impurity scattering, the edge for the transition involving chiral Landau level will be enhanced and the peaks for inter-band transitions will be suppressed ${ }^{27}$ (see details in Supplementary Note XV). Thus, it is reasonable to expect that the peak due to the transition edge has a similar feature with comparable amplitude as the feature from inter-band transitions. Secondly, the energy of the normal Landau level transitions, for example $\mathrm{T} 1-\mathrm{T} 7$, is determinedly by the distance between Landau levels at $k_{z}=0$, which is proportional to $\sqrt{B}$. Varying the Fermi level can only allow or abandon certain optical transitions, but it cannot affect the transition energy. For the linearly dispersed chiral Landau level, however, the transition edge energy is determined by the Fermi level as well, resulting in non- $\sqrt{B}$ field evolution. Thirdly, to observe the optical feature of the zeroth Landau level transition, it requires the system near the quantum limit (i.e., low-carrier-density sample under high magnetic fields). For the system far from that, the transition energy rapidly decreases to a vanishing value beyond our experimental resolution (Supplementary Note VI and Supplementary Figure 6, the experimental challenge of probing chiral Landau level). Fourthly, the optical behavior involving the zeroth Landau levels can be significantly influenced as the system approaches the quantum limit. Here, in our field regime, one of the Weyl nodes does not approach the quantum limit. Therefore, the Landau level intersects with the first Fermi level resulting in three intra-band transition edges as displayed in Fig. 3e. The upper two have the exactly same energy (Supplementary Note VIII). For those two transitions at same $k_{z}$, the lower one is the transition between chiral Landau level and $1^{\text {st }}$ Landau level while the upper one is the transition between $1^{\text {st }}$ and $2^{\text {nd }}$ Landau level. Further increasing the magnetic field enlarges the transition energy (Fig. 3d, red line). As long as the system reaches the quantum limit (Fig. 3f), the right edges will disappear since the intersection between Fermi level and $L_{1}$ no longer exists. The behavior of C3 transition fits well to the discussed scenario and the peak disappears near $12 \mathrm{~T}$.

Next, we performed a quantitative study for these intra-band transitions. Previously, the first-principle calculations reveal that $\mathrm{NbAs}$ is particle-hole asymmetric ${ }^{30}$. To capture the effect of particle-hole asymmetry in the massless system, we can add a diagonal term to the low-energy Hamiltonian that describes the Weyl fermions with different Fermi velocity on conduction and 
a

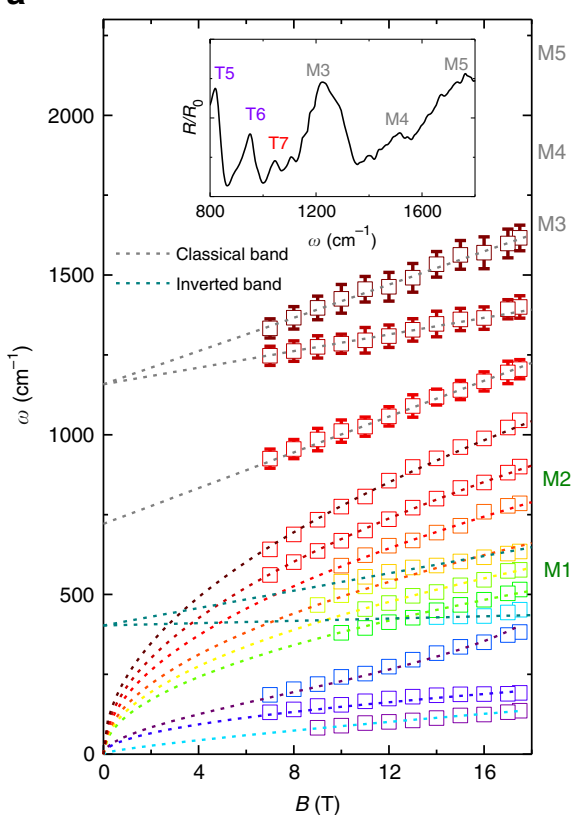

b
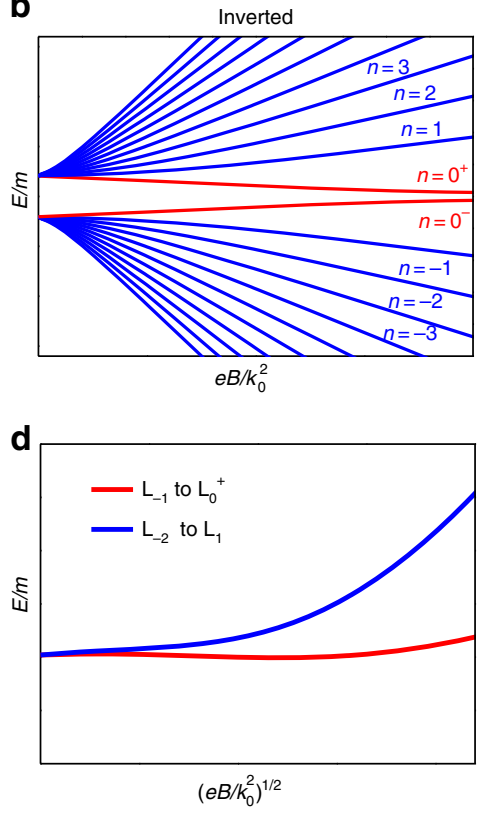

C
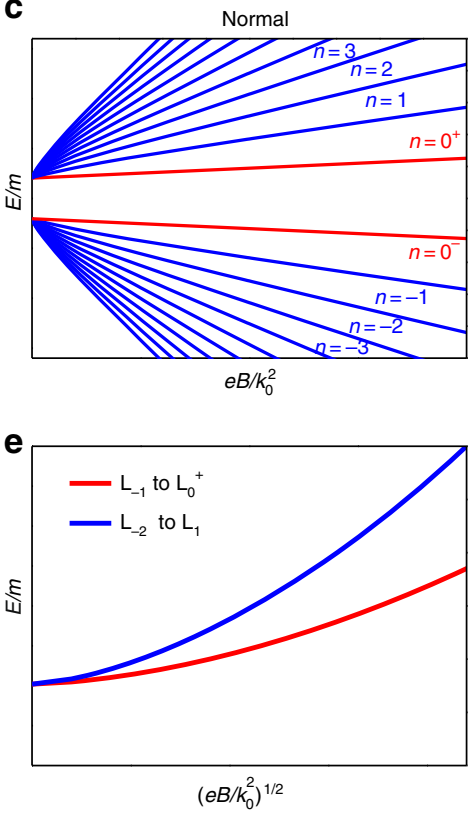

Fig. 4 Landau fan diagram of massive fermions with different topological nature. a Inter-Landau-level resonance energy versus magnetic field. For those transition already plotted in Fig.2a, error bars are not shown. Dashed straight lines with labels represent massive fermions with band gap. Color in dark green denotes the transition from the inverted gap structure. Color in gray describes the transitions from quadratic normal bands or undefined bands. The inset is the mid-infrared magneto-optic spectrum at $17.5 \mathrm{~T}$. b, c The Landau fan diagrams of the inverted and normal gap case according to Eq. (7), respectively. $\mathbf{d}$, e The frequency versus $\sqrt{B}$ relation of the lowest two optical transitions in $\mathbf{b}$ and $\mathbf{c}$, respectively. The parameters in Eq. (7) chosen for illustration are: for $\mathbf{b}$ and $\mathbf{d} C m / v^{2}=0.5, \Delta / m=0.5$; for $\mathbf{c}$, e $C m / v^{2}=-0.5, \Delta / m=0.5 ; k_{0}=\sqrt{m / 2 C}$. Note that the qualitative difference between the inverted case and normal case does not depend on the parameters chosen

valence band ${ }^{30}$ by

$$
H_{\alpha}(\mathbf{k})=\lambda_{\alpha} \sqrt{\eta_{1}^{2} k_{x}^{2}+\eta_{2}^{2} k_{y}^{2}+\eta_{3}^{2} k_{z}^{2}}+v_{\alpha} \mathbf{k} \cdot \sigma
$$

where $\alpha=1,2$ corresponds to the Weyl node reaching or not reaching the quantum limit, and $\lambda_{\alpha}$ denotes the effect of asymmetry. For $\lambda_{\alpha} \neq 0$, the conduction and valence bands are asymmetric. For simplicity, we assume $\eta_{1}=\eta_{2}=1$ and $\eta_{3}=0$. Similar to the previous derivation of Landau level, we consider that a magnetic field is exerted in $z$ direction, then the Landau level reads

$$
\begin{aligned}
& E_{n, \alpha}\left(k_{z}\right)=\left\{\begin{array}{l}
\lambda_{\alpha} f_{1}(n, B)+\operatorname{sgn}(n) v_{\alpha} \sqrt{\left(k_{z}-f_{2}(n, B)\right)^{2}+2 e B|n|},|n| \geq 1 \\
\lambda \sqrt{e B}-v_{\alpha} k_{z}, n=0
\end{array}\right. \\
& f_{1}(n, B)=(\sqrt{(2 n+1) e B}+\sqrt{(2 n-1) e B}) / 2, f_{2}(n, B) \\
& =\lambda_{\alpha}(\sqrt{(2 n+1) e B}-\sqrt{(2 n-1) e B}) / 2 v_{\alpha}
\end{aligned}
$$

As shown in Supplementary Note VII, the frequency influenced by the asymmetry is always orders of magnitude lower for the inter-band transition frequency. However, the effect on the intra-band transition cannot be neglected. We define the energy of the low (the left arrow in Fig. $3 \mathrm{e}$ and the arrow in Fig. 3f) and high (the bottom-right arrow in Fig. 3e) transition edges as $\omega_{0} \rightarrow 1,1$ and $\omega_{0 \rightarrow 1,2}$. For $\omega_{0 \rightarrow 1,1}$, the Fermi level intersects with chiral Landau level, therefore $\omega_{0 \rightarrow 1,1}=E_{1, \alpha}-\left|E_{\mathrm{F}}\right|_{v_{\alpha} k_{z}=\lambda_{\alpha} \sqrt{e B}-\left|E_{\mathrm{F}}\right|}$ can be calculated. For the high energy edge $\omega_{0 \rightarrow 1,2}$ from the Weyl node not in the quantum limit, the Fermi level intersects with the first Landau level. In the same manner, $k_{z}$ is firstly determined and then $\omega_{0 \rightarrow 1,2}=\left|E_{\mathrm{F}}\right|-E_{0, \alpha}$ can be calculated. Using the similar approach for the first and second Landau levels, we can calculate $\omega_{1-2}$ for T1 as well. The energy of the transition edge becomes a function of $E_{\mathrm{F}}, v, \lambda, B$. All details including the derivation of equations and data fitting are available in Supplementary Note VII. For a given band parameter, the position of Fermi level determines the quantum limit. Therefore, $E_{\mathrm{F}}$ is no longer a free parameter for the data fitting. C2 transition can be best fitted only by higher energy edge $\omega_{0 \rightarrow 1,2}$ as shown in Figs. 2a and 3d (fitting curves plotted as red solid line), which means that C2 is originated from the chiral Landau level in W1 (not in quantum limit). The fitting parameters are $v^{\prime} 1=2.2 \times 10^{5} \mathrm{~m} / \mathrm{s}$ and $\lambda_{1}=$ $1.1 \times 10^{5} \mathrm{~m} / \mathrm{s}$ which agree well with the previously-discussed origin of W1. When putting $v_{1}^{\prime}, \lambda_{1}$ into $\omega_{0 \rightarrow 1,1}$ and attempting to extract the position of the lower edge from W1, the calculated curve is almost overlapping with $\mathrm{T} 1$ as shown in Figs. $2 \mathrm{a}$ and $3 \mathrm{~d}$ (red solid line).

For $\mathrm{C} 1$ from $\mathrm{W} 2$, the best fit for $\omega_{0 \rightarrow 1,1}$ gives $v^{\prime}{ }_{2}=1.7 \times 10^{5} \mathrm{~m} / \mathrm{s}$ and $\lambda_{2}=0.26 \times 10^{5} \mathrm{~m} / \mathrm{s}$. The fitted curve is plotted as lower blue solid line in Fig. 3d. By examining the slope of T1 and $\omega_{1-2}$, the particle-hole asymmetry parameter is determined to be $\lambda_{1}{ }^{\prime}=1.0 \times 10^{5} \mathrm{~m} / \mathrm{s}$. Note that the equations in tables and figures use the Weyl model without particle-hole asymmetry for simplicity (see more details in Supplementary Note VIII). Using the fitted parameters from W2, one can predict the positions of higher frequency edge $\omega_{0 \rightarrow 1,2}$ from W2 as shown by the upper blue lines in Fig. 3d. The experimentally extracted C3 peaks fit well to the predicted curve. Therefore, the C3 peak comes from the higher frequency edge transition $\omega_{0 \rightarrow 1,2}$ from W2. In both Weyl nodes, the frequency of higher edge increases faster with magnetic field than the lower edge. As also determined 


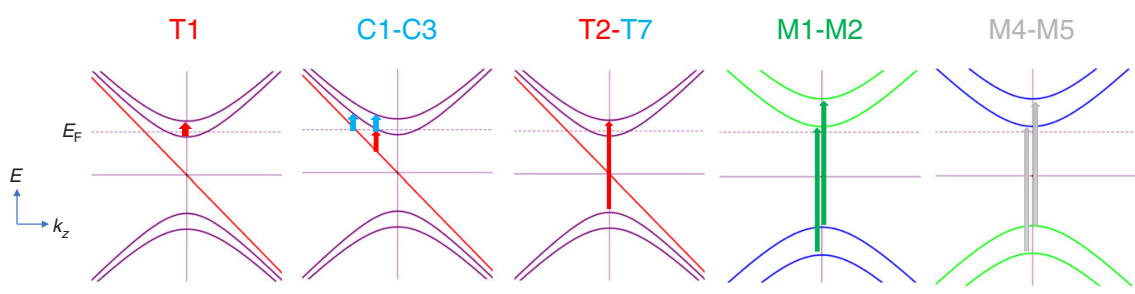

Fig. 5 Landau quantization of the quasi-particles summarized in NbAs. The energies of the Landau level are plotted versus $k_{z}$. Dashed lines are Fermi level. Arrows represent the allowed optical transitions. Red and blue colors denote the transition in Weyl node 1 and Weyl node 2, respectively. Green and gray colors represent the transitions in gapped electronic band with and without band inversion, respectively

\begin{tabular}{llllll} 
Table 1 Inter-Landau level transitions summarized in NbAs & & & \\
Optical transition & T1 & C1-C3 & T2-T7 & M1-M2 & M4-M5 \\
\hline Band & Weyl1 & Weyl1 \& Weyl2 & Weyl1 \& Weyl2 & Inverted gap & Topological trivial gap \\
Landau level dispersion & Normal & Linear \& chiral & Normal & Normal & Normal \\
Transition & Intra-band & Intra-band & Inter-band & Inter-band & Inter-band \\
$B$ evolution & $\sqrt{B}$ & $\pm \sqrt{c^{2} \pm B} \mp c$ & $\sqrt{B}$ & $\sim c$ & $B$
\end{tabular}

$B$ and $c$ denote the magnetic field and arbitrary constant, respectively. Note that for simplicity the equation for C1-C3 does not include particle-hole asymmetry

by the transport measurements, $\mathrm{W} 2$ reaches the quantum limit near $12 \mathrm{~T}$. Since the intersect between the Fermi level and the first Landau level is gone, it is reasonable to find that C3 peak disappears near $12 \mathrm{~T}$.

The fitting parameters $v, \lambda$ further verify the anticipated origin of the intra-band transitions accompanied by the following independent evidences: Firstly, experimental data points from all chiral Landau level transitions fit well with the magnetic field evolution as predicted by theory ${ }^{27,29}$ that clearly differs from the contribution of other bands; Secondly, the extracted velocity $v$ from $\mathrm{C} 1$ and $\mathrm{C} 2$ is very close to the value given by inter-band transitions where $v$ is acquired by $\sqrt{B}$ and $\sqrt{n}$ fitting. The edge behavior agrees well with our analysis for different situations (reaching the quantum limit or not); Thirdly, the extracted particle-hole asymmetry generally agrees with the features predicted by previous density functional theory (DFT) calculations ${ }^{30}$, where the Weyl node with higher Fermi velocity possesses a much stronger particle-hole asymmetry. Our conclusion of $\lambda_{1}>\lambda_{2}$ accords well with this prediction. The difference of Fermi velocity between particle and hole bands in previous DFT is $92 \%$ and $6 \%$ for W 1 and $\mathrm{W} 2^{30}$, respectively, while as a comparison our Landau level spectroscopy gives $53 \%$ and $15 \%$. With such a quantitative analysis, we indeed verify the existence of the chiral Landau level and particle-hole asymmetry in NbAs. To quantitatively describe all 10 features $(\mathrm{C} 1-\mathrm{C} 3$ and $\mathrm{T} 1-\mathrm{T} 7)$ in the spectra, it requires only two parameters of $v$ and $\lambda$ with a simple Weyl particle picture as described by Eq. (4).

\section{Discussion}

Other than C/T-type transition, there exist several M-type transitions at higher energy (also see the mid-infrared spectra in Supplementary Note IX and Supplementary Figure 7), which follow linear-in- $B$ evolution and clearly possess positive intercepts as shown in Fig. 4a. These features usually indicate a gapped structure corresponding to massive fermions ${ }^{50,51}$ that could coexist with Weyl band. Distinct from M4-M5 transitions in Figs. $2 \mathrm{a}$ and $4 \mathrm{a}$ where the slope ratio between the adjacent ones is 2:1 (reasonable in classical quadratic band). However, a very large slope ratio is found between M1 and M2. To understand this, we have provided the main physics of an IS-breaking Weyl semimetal by a four-band minimal model whose form takes

$$
H(k)=v_{x} k_{x} \tau_{x} \sigma_{z}+v_{y} k_{y} \tau_{y}+v_{z} k_{z} \tau_{x} \sigma_{x}+\left(m-C k^{2}\right) \tau_{z}+\Delta \tau_{y} \sigma_{x}
$$

where $v_{x, y, z}$ represent the velocities, $m$ and $C$ are two constants, and $\Delta$ denotes the IS-breaking term. With the introduction of magnetic field in $z$ direction, the Landau levels corresponding to the two gapped bands follow

$$
E_{n}\left(k_{z}\right)=\left\{\begin{array}{c}
\operatorname{sgn}(n) \sqrt{v^{2} k_{z}^{2}+\left(\sqrt{2 v^{2}|n| e B}+|\Delta|\right)^{2}+m\left(n, k_{z}\right)^{2}},|n| \geq 1 \\
\pm \sqrt{v^{2} k_{z}^{2}+\Delta^{2}+m\left(0, k_{z}\right)^{2}},|n|=0 .
\end{array}\right.
$$

where $m\left(n, k_{z}\right)=m-C k_{z}^{2}-2 e B C\left(|n|+\frac{1}{2}\right)$, and here we have assumed $v_{x}=v_{y}=v_{z}$ for simplicity. From topological insulators, we understand that the band inversion is the key to the realization of many exotic topological phases ${ }^{44}$ and the nearly zero slope feature of the M1 transition indicates the transition energy does not change much with magnetic field, which suggests the identical change of the initial and final Landau levels. Thus, it is of great significance to distinguish the inverted band structure from the normal band structure in experiments. The corresponding Landau fan diagrams are shown in Fig. 4 b, c, where the zeroth Landau levels for the normal case and inverted case are quite different. For the normal case with quadratic dispersion (Fig. 4c), the energies of all Landau levels in particle (hole) branch increase (decrease) monotonically with the magnetic field and the slope ratio of transition $L_{-1} \rightarrow L_{0}$ and $L_{-2} \rightarrow L_{1}$ should be around 2 . However, for the inverted case, the energy of the zeroth Landau level always shows an opposite monotonic behavior to other Landau levels belonging to the same branch. Consequently, the lowest spectrum corresponding to the optical transition $L_{-1} \rightarrow$ $L_{0^{+}}$or $L_{0^{-}} \rightarrow L_{1}$ can be very flat in a certain magnetic field regime leading to a large $\mathrm{M} 2 / \mathrm{M} 1$ ratio. Based on the theoretical calculations, the spectra in Figs. $2 \mathrm{a}$ and $4 \mathrm{a}$ with finite zero-field intercepts 
can be understood. M1 and M2 are from a gapped band with band inversion. As shown by Fig. 4c, e and Supplementary Note XI, M4 and M5 are originated from normal (non-inverted) bands. The overall understanding of the Landau quantization from multiple bands are summarized in Fig. 5 and Table 1.

Here we provide a general understanding of zeroth Landau levels in topological materials. In two-dimensional Dirac semimetals, such as graphene or the surface state of three-dimensional topological insulators, the lower dimension makes Landau levels (including the zeroth one) non-dispersed along $k_{z}$ directions. Therefore, a gap will be generated between adjacent Landau levels. This effect is most prominent between the zeroth and the first Landau level. The transition energy thus follows $\sqrt{B}$. However, in conventional bulk materials, the zeroth Landau level is parabolically dispersed along $k_{z}$. Although the gap vanishes, density of state diverges at $k_{z}=0$, resulting in the resonant energy (between the zeroth and the first Landau level) following $B$. In the bulk state of topological insulator and the inverted gap state observed in NbAs, while the optical transition is still limited at $k_{z}=0$, the energy of the zeroth Landau level decreases with magnetic field due to the band inversion. In Weyl semimetal, the zeroth chiral Landau level is linearly dispersed in $k_{z}$, resulting in two distinct features: optical transition away from $k_{z}=0$; and the transition frequency and momentum position highly dependent on the Fermi level. The transition energy is a function of both magnetic field and Fermi level.

In conclusion, we have studied the quasiparticle dynamics with contributions from multiple bands in NbAs through magnetooptical spectroscopy. All the optical features are summarized in Fig. 2a, Fig. 5, Table 1, and Supplementary Note IV. Taking the advantage of probing Landau levels under high magnetic fields, unconventional optical transitions reveal the existence of the zeroth chiral Landau level. Different from other degenerated Landau levels with non-zero index, it unconventionally disperses linearly in $k_{z}$ direction and allows the optical transition without the limitation of $k_{z}=0$ or $\sqrt{B}$ evolution. Finite scattering helps to enhance the feature from chiral Landau level. Besides, two inequivalent Weyl nodes are found with different Fermi velocity. Fitting the magnetic field dependence of optical transitions unveils that the Weyl node in NbAs with higher Fermi velocity possesses much stronger particle-hole asymmetry. Massive Dirac fermions and massive trivial fermions are also found by observing the linear-in- $B$ cyclotron resonance. We summarize the overall Hamiltonian in Supplementary Note XIII and also provide a general picture of the zeroth Landau levels in topological materials. A comparison of the Landau quantization between Weyl fermions and massive fermions was made to further verify the unique feature of the zeroth chiral Landau levels.

\footnotetext{
Method

Sample growth. NbAs single crystals were grown by enhanced chemical vapor transport method using iodine as the agent with tilted ampules angle to maximize the growth rate and crystal quality. A temperature gradient is required to provide the necessary driving force for gaseous species in diffusion and connection between the cooler and hotter end. The stoichiometry and crystal structure were examined by XRD (Bruker D8 Discover) and EDX in a scanning electron microscopy.
}

\footnotetext{
Magneto-optical measurements. The far-infrared and mid-infrared reflection were measured in a Faraday configuration with a superconducting magnet up to 17.5 T. NbAs samples were exposed to the globar infrared light through light pipes with (001) surface perpendicular to both the incident light and magnetic field. Infrared light was focused with parabolic cone. The reflected light was detected by a bolometer and analyzed by a Fourier transform infrared spectrometer. All the light tube, samples and bolometer were kept at liquid helium temperature in a cryostat. The light path was pumped under vacuum to avoid the absorption of water and other gases.
}

Zero-field reflection measurements. Zero-field reflection spectrum was measured with $E$ // (001) surface at liquid helium temperature on a combination of Bruke $113 \mathrm{~V}$ and $80 \mathrm{~V}$. An in-situ overcoating technique was used as reference.

Magneto-transport measurements. Magneto-transport measurements were carried out using physical property measurement system and pulsed magnetic field up to $60 \mathrm{~T}$ at Wuhan National High Magnetic Field Center. A standard Hall-bar geometry is made. Lock-in amplifiers were used to measure the electrical signals.

Data availability. The data that support the findings of this study are available from the corresponding author upon reasonable request

Received: 29 November 2017 Accepted: 4 April 2018

Published online: 10 May 2018

\section{References}

1. Weng, H., Fang, C., Fang, Z., Bernevig, B. A. \& Dai, X. Weyl Semimetal phase in noncentrosymmetric transition-metal monophosphides. Phys. Rev. X 5, 011029 (2015).

2. Lv, B. Q. et al. Experimental discovery of Weyl semimetal TaAs. Phys. Rev. X 5, 031013 (2015).

3. $\mathrm{Xu}, \mathrm{S}$. Y. et al. Discovery of a Weyl fermion semimetal and topological Fermi arcs. Science 349, 613-617 (2015).

4. Suzuki, T. et al. Large anomalous Hall effect in a half-Heusler antiferromagnet. Nat. Phys. 12, 1119-1123 (2016).

5. Ikhlas, M. et al. Large anomalous Nernst effect at room temperature in a chiral antiferromagnet. Nat. Phys. 13, 1085 (2017).

6. Liu, J. et al. A magnetic topological semimetal Sr 1-y Mn 1- z Sb 2 (y, z 0.1). Nat. Mater. 16, 905 (2017).

7. Huang, S. M. et al. A Weyl fermion semimetal with surface Fermi arcs in the transition metal monopnictide TaAs class. Nat. Commun. 6, 7373 (2015).

8. Weng, H., Dai, X. \& Fang, Z. Topological semimetals predicted from firstprinciples calculations. J. Phys. Condens. Matter 28, 303001 (2016).

9. $\mathrm{Xu}, \mathrm{S}$.-Y. et al. Discovery of a Weyl fermion state with Fermi arcs in niobium arsenide. Nat. Phys. 11, 748-754 (2015).

10. $\mathrm{Xu}, \mathrm{N}$. et al. Observation of Weyl nodes and Fermi arcs in tantalum phosphide. Nat. Commun. 7, 11006 (2016).

11. $\mathrm{Xu}, \mathrm{S}$. Y. et al. Experimental discovery of a topological Weyl semimetal state in TaP. Sci. Adv. 1, 40-43 (2015).

12. Yang, L. X. et al. Weyl semimetal phase in the non-centrosymmetric compound TaAs. Nat. Phys. 11, 728-732 (2015).

13. Lv, B. Q. et al. Observation of Weyl nodes in TaAs. Nat. Phys. 11, 724-727 (2015).

14. Sun, Y., Wu, S.-C. \& Yan, B. Topological surface states and Fermi arcs of the noncentrosymmetric Weyl semimetals TaAs, TaP, NbAs, and NbP. Phys. Rev. B 92, 115428 (2015).

15. Moll, P. J. et al. Magnetic torque anomaly in the quantum limit of Weyl semimetals. Nat. Commun. 7, 12492 (2016).

16. Son, D. T. \& Spivak, B. Z. Chiral anomaly and classical negative magnetoresistance of Weyl metals. Phys. Rev. B 88, 104412 (2013).

17. Liu, C.-X., Ye, P. \& Qi, X.-L. Chiral gauge field and axial anomaly in a Weyl semimetal. Phys. Rev. B 87, 235306 (2013).

18. Nielsen, H. B. \& Ninomiya, M. Adler-Bell-Jackiw anomaly and Weyl fermions in crystal. Phys. Lett. 130, 389-396 (1983).

19. Huang, $X$. et al. Observation of the chiral-anomaly-induced negative magnetoresistance in 3D Weyl semimetal TaAs. Phys. Rev. X 5, 031023 (2015).

20. Xiong, J. et al. Evidence for the chiral anomaly in the Dirac semimetal $\mathrm{Na}_{3} \mathrm{Bi}$. Science 350, 413 (2015).

21. Reis RDd et al. On the search for the chiral anomaly in Weyl semimetals: the negative longitudinal magnetoresistance. New J. Phys. 18, 085006 (2016)

22. Zhang, C.-L. et al. Signatures of the Adler-Bell-Jackiw chiral anomaly in a Weyl fermion semimetal. Nat. Commun. 7, 10735 (2016).

23. Zhang, C. et al. Room-temperature chiral charge pumping in Dirac semimetals. Nat. Commun. 8, 13741 (2017).

24. Li, Q. et al. Chiral magnetic effect in ZrTe5. Nat. Phys. 12, 550-554 (2016).

25. Li, Y. et al. Negative magnetoresistance in Weyl semimetals NbAs and NbP: intrinsic chiral anomaly and extrinsic effects. Front. Phys. 12, 127205 (2016)

26. Armitage, N. P., Mele, E. J. \& Vishwanath, A. Weyl and Dirac semimetals in three-dimensional solids. Rev. Mod. Phys. 90, 015001 (2018).

27. Ashby, P. E. C. \& Carbotte, J. P. Magneto-optical conductivity of Weyl semimetals. Phys. Rev. B 87, 245131 (2013).

28. Nielsen, H. B. \& Ninomiya, M. The Adler-Bell-Jackiw anomaly and Wey fermions in a crystal. Phys. Lett. B 130, 389-396 (1983). 
29. Akrap, A. et al. Magneto-optical signature of massless kane electrons in $\mathrm{Cd}_{3} \mathrm{As}_{2}$. Phys. Rev. Lett. 117, 136401 (2016).

30. Lee, C.-C. et al. Fermi surface interconnectivity and topology in Weyl fermion semimetals TaAs, TaP, NbAs, and NbP. Phys. Rev. B 92, 235104 (2015).

31. Boller, H. \& Parthé, E. The transposition structure of NbAs and of similar monophosphides and arsenides of niobium and tantalum. Acta Crystallogr. 16, 1095-1101 (1963).

32. Li, Z. et al. Weyl semimetal TaAs: crystal growth, morphology, and thermodynamics. Cryst. Growth Des. 16, 1172-1175 (2016).

33. Furuseth, S. \& Kjekshus, A. The crystal structure of NbAs (comments). Acta Crystallogr. 17, 1077-1078 (2001).

34. Besara, T. et al. Coexistence of Weyl physics and planar defects in the semimetals TaP and TaAs. Phys. Rev. B 93, 245152 (2016).

35. Basov, D. N., Fogler, M. M., Lanzara, A., Wang, F. \& Zhang, Y. Colloquium: graphene spectroscopy. Rev. Mod. Phys. 86, 959-994 (2014).

36. $\mathrm{Li}, \mathrm{Z}$. Q. et al. Infrared probe of the anomalous magnetotransport of highly oriented pyrolytic graphite in the extreme quantum limit. Phys. Rev. B 74, 195404 (2006).

37. Kuzmenko, A. B. Kramers-Kronig constrained variational analysis of optical spectra. Rev. Sci. Instrum. 76, 775 (2005).

38. Lax, B. \& Mavroides, J. G. Chapter 8 interband magnetooptical effects. Semicond. \& Semimet. 3, 321-401 (1967).

39. Wang, Y. et al. Observation of ultrahigh mobility surface states in a topological crystalline insulator by infrared spectroscopy. Nat. Commun. 8, 366 (2017).

40. Jiang, Z. et al. Infrared spectroscopy of Landau levels of graphene. Phys. Rev. Lett. 98, 197403 (2007).

41. Orlita, M. \& Potemski, M. Dirac electronic states in graphene systems: optical spectroscopy studies. Semicond. Sci. Technol. 25, 063001 (2010).

42. Chen, R. et al. Magnetoinfrared spectroscopy of Landau levels and Zeeman splitting of three-dimensional massless Dirac fermions in $\mathrm{ZrTe}_{5}$. Phys. Rev. Lett. 115, 176404 (2015).

43. Yuan, X. et al. Observation of quasi-two-dimensional Dirac fermions in $\mathrm{ZrTe}_{5}$. NPG Asia Mater. 8, e325 (2016).

44. Hasan, M. Z. \& Kane, C. L. Colloquium: topological insulators. Rev. Mod. Phys. 82, 3045-3067 (2010).

45. Ghimire, N. J. et al. Magnetotransport of single crystalline NbAs. J. Phys. Condens. Matter 27, 152201 (2015).

46. Luo, Y. et al. Electron-hole compensation effect between topologically trivial electrons and nontrivial holes in NbAs. Phys. Rev. B 92, 205134 (2015).

47. Jiang, Y. et al. Landau-level spectroscopy of massive Dirac fermions in singlecrystalline ZrTe5 thin flakes. Phys. Rev. B 96, 041101 (2017).

48. Orlita, M. et al. Observation of three-dimensional massless Kane fermions in a zinc-blende crystal. Nat. Phys. 10, 233-238 (2014).

49. Li, G. \& Andrei, E. Y. Observation of Landau levels of Dirac fermions in graphite. Nat. Phys. 3, 623-627 (2007).

50. Yuan, X. et al. Direct observation of Landau level resonance and mass generation in Dirac semimetal Cd3As2 thin films. Nano Lett. 17, 2211-2219 (2017).

51. Orlita, M. et al. Magneto-optics of massive dirac fermions in bulk Bi2Se3. Phys. Rev. Lett. 114, 186401 (2015).

\section{Acknowledgements}

This work was supported by the National Natural Science Foundation of China (Grant No. 11474058, 61674040, 11674189) and the National Key Research and Development Program of China (Grant No. 2017YFA0303302, 2016YFA0203900, 2017YFA0303504). We thank Ying Wang, Xue Jiang, Zhiqiang Li, and Nanlin Wang for helpful discussions. H.Y. is also grateful to the financial support from Oriental Scholar Program from Shanghai Municipal Education Commission. A portion of this work was performed at the National High Magnetic Field Laboratory, which is supported by National Science Foundation Cooperative Agreement No. DMR-1157490, DMR-1644779, and the State of Florida. Part of the sample fabrication was performed at Fudan Nano-fabrication Laboratory.

\section{Author contributions}

F.X. conceived the idea and supervised the experiments. Z.L. \& X.C. carried out the growth. X.Y., C.Z., C.S., W.W., Y.L., J.L., D.S., T.X. and H.Y. performed the magnetooptical experiments. C.S. and H.Y. did magneto-Drude-Lorentz fitting. M.Z. carried out the zero-field reflection measurements. X.Y., C.Z., Y.L., X.Z. and Z.X. conducted magneto-transport experiments. Z.Y. and Z.W. performed theoretical calculations. X.Y., Z.Y., C.Z. and F.X. wrote the paper with help from all other authors.

\section{Additional information}

Supplementary Information accompanies this paper at https://doi.org/10.1038/s41467018-04080-4.

Competing interests: The authors declare no competing interests.

Reprints and permission information is available online at http://npg.nature.com/ reprintsandpermissions/

Publisher's note: Springer Nature remains neutral with regard to jurisdictional claims in published maps and institutional affiliations.

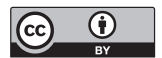

Open Access This article is licensed under a Creative Commons Attribution 4.0 International License, which permits use, sharing, adaptation, distribution and reproduction in any medium or format, as long as you give appropriate credit to the original author(s) and the source, provide a link to the Creative Commons license, and indicate if changes were made. The images or other third party material in this article are included in the article's Creative Commons license, unless indicated otherwise in a credit line to the material. If material is not included in the article's Creative Commons license and your intended use is not permitted by statutory regulation or exceeds the permitted use, you will need to obtain permission directly from the copyright holder. To view a copy of this license, visit http://creativecommons.org/ licenses/by/4.0/.

(C) The Author(s) 2018 